Introduction The National Institute of Clinical Excellence (NICE) in the United Kingdom (UK) approved the use of Radiofrequency ablation (RFA) as minimally invasive endoscopic therapy for the treatment of Barrett's Oesophagus related neoplasia as an alternative to surgery in 2010. These high risk patients carry a 40-60\% risk of progressing to Osophageal Adenocarcinoma (OAC), survival from which is poor. Over the past 5 years combined endoscopic mucosal resection (EMR) and RFA have become the preferred intervention for the curative treatment of patients with BE related neoplasia.

Methods We report prospective data from one of the UK's largest academic tertiary centres for patients undergoing RFA for early neoplasia arising in BE between 2008-2013 at University College Hospital, London. Before RFA, visible lesions were removed by EMR. Patients then underwent RFA every 3 months until all visible BE was ablated or cancer developed (endpoints). Biopsies were taken at 12 months or when endpoints reached. Primary outcomes were clearance for dysplasia (CR-D) and BE and intestinal metaplasia (CR-IM) at 12 months. Long term durability for CR-D for those with favourable outcomes at 12 months was assessed.

Results Two hundred patients have undergone RFA since 2007 at our centre. Of these 145 have completed treatment. Most are male (83\%), mean age 69 years (range 44-91). Baseline histology HGD in $86 \%$ and IMC in $14 \%$. Mean length BE segment prior to RFA $6 \mathrm{~cm}$ (range 1-20). Ten per cent patients in our cohort underwent RFA for residual neoplasia after unsuccessful Photodynamic therapy (PDT). Prior to RFA treatment, 50\% of patients had EMR for visible lesions. After 2-3 RFA treatments (range 1-6) over 12 months, 80\% (116/45) patients had achieved CR-D and 65\% CR-IM (94/145). Pre-treatment PDT, EMR or histology did not influence outcomes. Three patients $(2.5 \%)$ progressed to invasive OAC at 12 months and in total 8 (7\%) progressed at most recent follow up. At 5 years $95 \%$ of patients who had dysplasia clearance at 12 months remain disease free (median follow up 20 months, IQR 9-32). Kaplan Meir survival statistics demonstrate at 5 years after successful treatment $75 \%$ and $77 \%$ of patients are likely to remain free of dysplasia and IM respectively.

Conclusion We report on the UK's single largest prospective series to date of patients undergoing endoscopic therapy for $\mathrm{BE}$ related neoplasia. Our outcomes compare favourably with those published around the world and from within the UK patient registry. These patients are high risk and require vigilant follow up even after successful treatment as predicted recurrence can occur in up to $25 \%$ of cases.

Disclosure of Interest None Declared.

\section{PTU-041 SUB-SQUAMOUS COLUMNAR NEOPLASIA AFTER SUCCESSFUL RADIOFREQUENCY ABLATION FOR BARRETT'S RELATED NEOPLASIA IS RARE BUT HIGHLIGHTS REQUIREMENT FOR LONG TERM FOLLOW UP IN THESE PATIENTS}

${ }^{1,2}{ }^{2}$ J Haidry ${ }^{*},{ }^{1} \mathrm{M}$ Banks, ${ }^{1} \mathrm{~A}$ Gupta, ${ }^{2} \mathrm{M}$ Butt, ${ }^{1} \mathrm{M}$ Rodriguez-Justo, ${ }^{1} \mathrm{M}$ Novelli, ${ }^{1,2} \mathrm{~L}$ Lovat. ${ }^{1} U C L H$, London, UK; ${ }^{2} N M L C, U C L$, London, UK

\subsection{6/qutjil-2014-307263.115}

Introduction Radiofrequency ablation (RFA) combined with endoscopic mucosal resection (EMR) has become the preferred treatment for BE related neoplasia. Success rates of $90 \%$ after treatment with a durability of up to $95 \%$ at 5 years are reported. The development of sub-squamous intestinal metaplasia (IM) after successful RFA is recognised and has been reported to range from $0-30 \%$. It's clinical significance remains unclear. However the development of sub-squamous neoplasia after successful treatment is limited to only a few cases world wide in the literature.

Methods We prospectively examine the incidence of sub-squamous neoplasia after successful RFA from one of the UKs largest academic tertiary centres for patients undergoing RFA for BE neoplasia between 2008-2013 at University College Hospital, London. Before RFA, visible lesions and nodularity were entirely removed by EMR. Thereafter patients underwent RFA every 3 months until all visible $\mathrm{BE}$ was ablated. Biopsies were taken at 12 months. After successful treatment patients were followed up 3 monthly for the first year, at 6 month intervals for the second year and annually thereafter. Biopsies were taken from $1 \mathrm{~cm}$ below the neo z-line and from the previously treated BE segment using the Seattle protocol. Enhanced imaging endoscopic imaging was used in all cases.

Results At our institution 197 patients have undergone RFA since 2007 of which 145 have completed treatment. Eighty percent of patients (116/145) achieved CR-D at 12 months. At 5 years $90 \%$ of patients remain disease free (median follow up 20 months, IQR 9 -32). There have been a total of 11 recurrences after successful RFA (median time to recurrence - 380 days, IQR 150-884). Four of these patients had sub-squamous high grade glandular dysplasia (HGD). All had had confirmed eradication of dysplasia and IM after the 12 month end of protocol biopsy. These sub-squamous recurrences occurred at 3, 7, 13 and 36 months respectively after eradication was confirmed. One of the four patients was found to have buried HGD on biopsy at the neo z-line and the area was subsequently treated with EMR. The other 3 cases developed visible lesions in neosquamous mucosa proximal to the neo z-line. All were removed successfully with EMR and demonstrated buried HGD. All 4 cases remain in follow up with no neoplasia at most recent biopsy.

Conclusion Although rare, buried neoplasia after successful eradication with RFA can occur. Identification of these cases indicates the need for continued and vigilant surveillance following RFA, even after complete eradication of IM. Early recognition of recurrence can lead to treatment at an early stage.

Disclosure of Interest None Declared.

\section{PTU-042 APPROPRIATELY TRAINED REGISTRARS ARE AS GOOD AS CONSULTANTS IN ENDOSCOPIC MANAGEMENT OF MODERATE-SEVERE UPPER GASTROINTESTINAL HAEMORRHAGE}

M McShane, R Hewett*, G Sadler, A Poullis. Gastroenterology, St George's Healthcare NHS Trust, London, UK

\subsection{6/gutjnl-2014-307263.116}

Introduction The South West Thames region operates a unique regional gastrointestinal service covering 4 hospitals. Registrar endoscopists are assessed and signed off as competent in providing endoscopic haemostatic therapy before taking their place on the GI bleed rota, and are supported by an on-call consultant. We aimed to evaluate if there were any operator-dependent differences in outcomes of endoscopic procedures performed in cases of moderate-severe upper gastrointestinal haemorrhage between consultants and registrars

Methods Data were recorded for all emergency OGDs performed at St. George's Hospital, between 01/01/13 and 30/06/ 


\begin{tabular}{llll} 
Abstract PTU-042 & Table 1 & & \\
\hline & & Consultant-led & \\
Rockall score & (Mortality \%) & OGD \\
(Mortality \%) & p value \\
\hline 1 & 0 & 0 & n.s. \\
2 & 0 & $*$ & n/a \\
3 & 27.27 & 25 & n.s \\
4 & 35.71 & 12.5 & n.s \\
5 & 25 & 57.14 & n.s \\
6 & 40 & 100 & n.s \\
7 & 42.86 & 0 & n.s. \\
8 & 66.67 & * & n/a \\
\hline *No consultant-led procedures with this Rockall score in the sample. &
\end{tabular}

13 , in patients presenting with haematemesis or melaena and requiring endoscopy. The Rockall score for each patient was calculated and plotted against mortality for the whole population, and then sub classified into consultant- or registrar-performed procedures.

Results 110 procedures performed on 101 patients (41 female, 60 male, median age 71 , age range $23-93$, and an overall mortality of $29.7 \%$ ) were analysed

A direct relationship between an increasing Rockall score and mortality was demonstrated in both the overall population, and also in the registrar-performed procedures, inferring typical population characteristics. Mortality for registrar-performed procedures showed no appreciable difference to that of the overall patient population.

Conclusion Descriptors for achieving competence in therapeutic endoscopy have yet to be defined by the JAG. However, provided local mechanisms for delivering training in, and assessment of, competence at providing endoscopic haemostatic therapy are robust, our findings support the use of a registrar-led out-ofhours GI bleed service.

Disclosure of Interest None Declared.

\section{PTU-043 CAPSULE ENDOSCOPY RETENTION; IS PREDICTION WITHOUT A PATENCY PRE-STUDY POSSIBLE?}

${ }^{1}$ R Makins*, ${ }^{2} \mathrm{R}$ Hopkins. 'Gastroenterology, Cheltenham General Hospital, Cheltenham, UK; ${ }^{2}$ Radiology, Cheltenham General Hospital, Cheltenham, UK

\subsection{6/gutjnl-2014-307263.117}

Introduction Small intestinal capsule endoscopy (CE) is used to investigate occult gastrointestinal bleeding, iron deficiency anaemia, to evaluate the small intestine for Crohn's disease (CD), polyposis syndromes or complications of NSAID use. The only significant complication is capsule retention, either long term or transient, potentially leading to luminal obstruction. The Patency Capsule can be used prior to CE to confirm the likelihood of safe passage however is only for use in conjunction with the system by Given Imaging (Diagmed UK, Personal communication). We use Mirocam CE equipment (Intromedic). Consequently reliance must be placed on radiological studies along with clinical evaluation when assessing the risk of retention. The aim of this study was to determine, in patients where capsule retention had occurred in the setting of a previously reported normal radiological study, whether retrospective review of the radiology could identify stricturing disease and therefore determine whether retention could have been predicted with prior radiological imaging alone.
Methods We reviewed records of all patients who had undergone CE in Gloucestershire since the inception of the local service in 2008. Identified all patients in whom capsule retention occurred. Defined capsule retention as occuring in patients where a stricturing lesion was seen, the capsule appeared not to pass the lesion and colonic mucosa was not positively identified. We retrospectively reviewed any relevant radiological studies performed prior to the CE to determine whether, with hindsight, abnormalities could be identified that would have significantly increased the risk of capsule retention.

Results 465 CE performed locally in Gloucestershire since 2008. CE retention occured above a stricture in 6 (4 male, mean age 45, range 27-61). Indications for CE abdominal pain +/anaemia. In 5 the strictures were thought to be secondary to $\mathrm{CD}$ (past history of CD in 2 only), in 1 due to NSAID use. No stricturing lesions had been identified previously on either barium follow through (2) or cross sectional (CT) imaging (4). 4 studies had been performed locally, 2 by other providers, reported by non-specialist radiologists. On retrospective review significant stricturing lesions were seen on all studies, findings contraindicating CE.

Conclusion $\mathrm{CE}$ is a safe and widely used tool. The risk of capsule retention increases in the setting of Crohn's disease. We have shown that in cases where capsule retention occurred in our institution, where prior radiology was reported as normal, stricturing lesions could be positively identified when reviewed retrospectively by a specialist radiologist. We conclude that thorough radiological assessment is adequate to predict capsule retention and patency studies prior to $\mathrm{CE}$ are therefore unnecessary.

Disclosure of Interest None Declared.

\section{PTU-044 TERTIARY CENTRE EXPERIENCE OF 360 DEGREE SIDE-VIEWING VIDEO CAPSULE ENDOSCOPY}

R Goel*, DM Borrow, KV Patel, I Nasr, M Ward, S Ray, PM Irving, JD Sanderson, SH Anderson. Gastroenterology, Guy's and St Thomas' NHS Foundation Trust, London, UK

\subsection{6/gutjnl-2014-307263.118}

Introduction Since it's development in 1999, video capsule endoscopy (VCE) has become the investigation of choice for examining the small bowel. Recently, a novel panoramic 360 degree side-viewing VCE (Capsovision, Medical Innovations, USA) was launched. It differs from previous capsules in that no data recorder or sensors are required. The images are stored on the capsule itself, which when passed, must be retrieved and sent to the endoscopy reader for analysis.

We report our initial experience of this novel VCE.

Methods We retrospectively analysed the first 51 side-viewing VCE over a 6-month period at our institution. All patients had a clear liquid diet as preparation the day before.

Results 51 patients (26 males) underwent examination with the side-viewing VCE.

39 (76.4\%) examinations were completed and 12 were incomplete. This included 4 which were lost due to being flushed away. Over the same time period, forward-viewing VCE complete results were available in $83.2 \%$ patients.

1 of the incomplete examinations was due to a NSAIDinduced stricture, subsequently diagnosed with a forward-viewing VCE.

31 patients had good bowel preparation, 11 satisfactory preparation and 6 were reported as having poor bowel preparation. 